\title{
EFFECTS OF VITAMIN D ON THE RENIN-ANGIOTENSIN SYSTEM
}

\section{Pashova-Stoyanova*, A. Tolekova}

\author{
Department of Physiology, Pathophysiology and Pharmacology, Medical Faculty, Trakia University, \\ Stara Zagora, Bulgaria
}

\begin{abstract}
The renin-angiotensin-aldosterone system (RAAS) is a complex endocrine system of enzymes, proteins and peptides that occupies a key position in the regulation of a number of important physiological processes, such as arterial pressure, water and electrolyte homeostasis. Its activity, flow and regulation are affected by a large number of mediators, substances and diseases one of which is vitamin D. Vitamin D is involved in the regulation of many physiological processes with great importance. Vitamin $\mathrm{D}$ deficiency is associated with an increased risk of impaired renal function, cardiovascular disease, diabetes mellitus, metabolic disorders, affecting RAAS and other pathways.
\end{abstract}

Key words: vitamin D, renin-angiotensin system

\section{INTRODUCTION}

The renin-angiotensin system is a unique regulatory system by its nature and characteristics. It has numerous effects on the regulation of a number of systems and mediators, and at the same time is influenced by the impact of a number of substances and factors.

Vitamin D is beneficial for the regulation of bone metabolism, calcium homeostasis and affects different aspects of people's health. There is evidence that vitamin D is important for normal blood pressure, sugar levels, insulin resistance, immune system, reproductive system and many more.

Vitamin D affects the main effector of the renin-angiotensin system on different levels and leads to changes in the regulation of different mediators.

\section{Renin-angiotensin system}

The renin-angiotensin system has a key role in salt balance and cardiovascular homeostasis. Validated as a major humoral regulator of

\footnotetext{
*Correspondence to: Lilia Pashova-Stoyanova, Bulgaria, Stara Zagora, Trakia University, Medical Faculty, Armeiska 11 str, Department of Physiology, Pathophysiology and Pharmacology corresponding author:, e-mail: lils@gbg.bg,Tel: 0897427123
}

blood pressure and intravascular volume, the system is the subject of detailed and thorough research. Following the discovery of renin by Robert Tigerstedt in 1897, a new era in the development of physiology, biochemistry of peptide hormones, the pathophysiology of the hypertonic disease, endocrinology, pharmacology and a number of scientific research inquiries and angiotensins were launched (1-7).

The renin-angiotensin-aldosterone system (RAAS) is a complex endocrine system of enzymes, proteins and peptides that occupies a key position in the regulation of a number of important physiological processes, such as arterial pressure, water and electrolyte homeostasis (8-11).

The activity of RAAS is determined by the velocity of renin secretion by the juxtaglomerular apparatus of the kidney (12, 13).

Touyz (2002) points out that Its activity, flow and regulation are affected by a large number of diseases in connection with the onset of humoral, metabolic and other changes. Knowledge of the pathophysiological features, pathogenesis and mechanisms for the development of these disabilities helps to understand the disease thoroughly and to create 
specific, targeted and effective ways to counteract (14).

Rahimi et al (2014) suggest that there is a close relationship between the major RAAS effector, namely angiotensin II, insulin resistance, the progression of diabetic nephropathy, retinopathy, neuropathy, and cardiovascular lesions in patients with type 2 diabetes. There is also a close association between RAAS and the development of metabolic disorders, metabolic syndrome and its components and complications.

Studies by Agarwal (2003) and Arendshorts (1999) report that inclusion of drugs from the angiotensin-converting enzyme (ACE) inhibitors and angiotensin receptor blockers (sartans) in the treatment of patients with metabolic syndrome, pre-diabetic conditions and type 2 diabetes significantly reduces the risk of new diabetes, reduces proteinuria and reduces the progression of nephropathy to terminal renal disease, which supports the thesis of a link between RAAS and metabolic parameters (15-17).

\section{Vitamin D: role, effect, physiological importance}

Vitamin $\mathrm{D}$ belongs to the family of the fatsoluble vitamins and can be found under different names, including anti-rheumatic vitamin, calciferol and others. Vitamin D discovery and isolation initiate in-depth studies of its role in the body, biological functions and reactions in the cases of deficiency $(18,19)$.

According to Heaney (2008) and Holick (2007) vitamin D is available in several different forms, from D1 to D5, each exhibiting different biological activity and functions. Only two of the forms are found in nature, namely vitamin D2, also known as ergocalciferol and vitamin D3, also called cholecalciferol. Ergocalciferol is essentially derived from ergosterin contained in wheat, irradiated with ultraviolet rays and is inactive vitamin D. Ergocalciferol is taken by food, and after subcutaneous deposition under the influence of ultraviolet rays, it is transformed into active vitamin D2 which rapidly is absorbed into the blood and can be stored for several months in the adipose tissue. The real anti-rheumatic vitamin is cholecalciferol or vitamin D3 (20, 21).

Autier (2007) points out that vitamin D exerts a significant influence on the cells responsible for bone tissue synthesis (osteoblasts), providing calcium and phosphate ion deposition. According to Yilmaz (2012) it also participates actively in the processes of bone and dentin, and this property is the opposite of parathyroid hormone action (parathyroid hormone regulates the processes and activity of osteoclasts, which in turn are responsible for the degradation of bone tissue) $(22,23)$.

Studies by Sipahi (2017) show that vitamin D is involved in the absorption of calcium and phosphorus in the small intestine, and under its action a specific protein is formed on the surface of the small intestinal mucosa, known as calcium binding protein, responsible for calcium binding and transport via active transport through the walls of the small intestine (24).

There is evidence from studies by Sipahi (2017) and Atalay (2017) that in the kidneys vitamin $\mathrm{D}$ affects the phosphate ion reabsorption, increasing it and increasing its blood concentration (hyperphosphatemia) in the course of phosphorous exchange, and this effect is also opposed to the parathormone effect $(24,25)$.

Mostafa et al (2016) report that regardless of the source of supply to the body with vitamin D (food, sun exposure, additives), it is necessary to double pass the process of hydroxylation to obtain the active form of vitamin $\quad \mathrm{D}, \quad$ namely 1,25dihydroxycholecalciferol.

The author states that the first hydroxylation process takes place in the liver, forming 25hydroxycholecalciferol (25-OH-D3) or 25hydroxyvitamin D3. The second hydroxylation process takes place in the kidney to form the final active metabolite, namely 1.25 dihydroxycholecalciferol (1,25- (OH) 2-D3). Their distinctive and characteristic feature is faster and stronger effect than even vitamin D itself. In renal structures, the parathyroid hormone plays the role of a stimulator of particular importance in the conversion of 25 $(\mathrm{OH}) \mathrm{D}$ to $1,25-(\mathrm{OH}) 2-\mathrm{D} 3$.

The reactions of conversion of the starting vitamin $\mathrm{D}$ into its active metabolite are accomplished with the enzymes 25hydroxylase in the liver and 1-alphahydroxylase in the kidney, both of which belong to the family of cytochrome P450dependent steroidal hydroxylase. 
According to Zittermann (2003) there is a close interdependence and interrelation between parathyroid hormone and vitamin $\mathrm{D}$. The synthesis of its active form requires the presence of parathormone and the action of parathyroid hormone on bone tissue requires the active principle of vitamin D3 $(26,27)$.

Holick (2004) explains that 1,25-(OH) 2-D3 performs its calcium function by binding to the vitamin D receptor (VDR), which in turn binds to DNA sequences (VDREs) and regulates a large number of genes. Activation of VDR in intestines, bones, kidneys and parathyroid glands maintains a normal concentration of calcium and phosphorus and hence bone mineralization (28).

Evidence from Basit (2013) indicates that all cells of acquired immunity (monocytes, dendritic cells, B cells, T cells, NK cells) express VDR continuously or after immune stimulation and are sensitive to vitamin D activity. High levels of vitamin D inhibit dendritic cell maturation by reducing in the production of proinflammatory molecules (IL2, IL-12, IL-17, IL-23) and enhance the expression of IL-10, resulting in an increase in regulatory T-cells. Additional vitamin D is involved in regulating acquired immunity and regulating T-lymphocyte proliferation and function.

Vitamin D also exhibits anti-inflammatory activity by its effects on $\mathrm{T}$ - and $\mathrm{B}$ lymphocytes, dendritic cells, macrophages and cytokines emitted by them.

According to data from Basit (2013) and Heaney (2008) the physiological effect and importance of vitamin D can be characterized by its participation in a number of important processes for the human body:

- 25-hydroxycalciferol, together with parathyroid hormone, contributes to the optimal maintenance of blood calcium levels;

- Maintain optimal levels of phosphorus in the blood;

- Maintain normal cell growth and functioning;

- Regulation of growth and cellular functions of brain cells;

- Participation in the processes of maintaining optimal immune function: decreases levels of certain proinflammatory cytokines (TNF, IL6), increases levels of certain antiinflammatory cytokines (IL-10);

- Regulating secretion of parathyroid hormone;
- It improves insulin secretion from pancreatic Beta cells and improves insulin sensitivity (18, 20).

Numerous studies and experimental studies have shown the unambiguous and extremely important role of vitamin $\mathrm{D}$ in protecting against the development of metabolic disorders. Since metabolic damage has been associated with numerous factors and metabolic disorders have developed at different levels, it has been shown that the fatsoluble vitamin significantly improves lipid profile and blood sugar disorders, which are some of the initial and leading to the manifestation of the entire spectrum of a component of the metabolic syndrome.

Sibuh (2018) points out that in experiments with rats subjected to high fructose diet, a significant improvement in serum glucose and lipid profile after vitamin D supplementation was observed and described. Supplementation was associated with protective properties in terms of developing dyslipidemia and hyperglycemia (23, 24, 27-29).

\section{Relationship between vitamin $D$ and the renin-angiotensin system}

$\mathrm{Li}$ (2003) suggests that the spectrum of physiological effects of vitamin $\mathrm{D}$ goes beyond its role as one of the factors involved in the regulation of calcium-phosphorus homeostasis and bone metabolism. Vitamin D also has pleiotropic effects - it actively participates in cell differentiation processes, in immune activity, in the maintenance of vascular wall integrity and cardiomyocytes, has antiproliferative activity, affects insulin resistance, modulates renin-angiotensin system (30).

According to Li (2002) and Vaidya (2012) the general focus of relationships and interdependence between the renin-angiotensin system, calcium ions, and calcium-regulating hormones (parathyroid hormone, vitamin D) are juxtaglomerular cells and renal arteriolar cells.

Renin secretion is dependent on the rate of formation of cyclic adenosine monophosphate, the concentration of which in turn is related to the ratio of the activity of cell mediated adenylyl cyclase to calmodulin-activated phosphodiesterase. The increased intracellular concentration of calcium results in the suppression of the formation of cyclic adenosine monophosphate. Elevated calcium 
cation levels inhibit renin secretion by means of extracellular and intracellular mechanisms $(31,32)$.

In studies by Chiu (2004) and Chandel (2017) it was found that in impaired renal function and activity levels of 1,25- (OH) 2-D3 decreased progressively, causing hypovitaminosis, and this deficit was unlikely to be due to diet. Low levels of $25(\mathbf{O H})$ vitamin $\mathbf{D}$ in patients with chronic kidney disease are associated with a higher risk of rapid progression of the disease and higher mortality. The main modifiable risk factor for determining the overall prognosis in patients with chronic kidney disease is the degree of albuminuria. Even low to moderate elevations of albuminuria are associated with an increased risk of progression of status, end-stage disease, increased overall and cardiovascular mortality. Suppression of the renin-angiotensin system is an important factor in the treatment of proteinuria in these patients. Experimental data in this field show an intriguing relationship between vitamin $\mathrm{D}$ and albuminuria, as the vitamin inhibits the inflammation and components of the renin-angiotensin system. Oral administration of cholecalciferol and calciferol has been shown to reduce proteinemia in individuals with moderately impaired glomerular diabetic nephropathy (33, 34).

Vaidya (2012) points out that low blood vitamin $\mathrm{D}$ concentrations directly correlate with higher angiotensin II concentrations and increased plasma renin activity, with vitamin D deficiency being associated with increased renin-angiotensin system activity. For example, Basit and associates found in patients on hemodialysis with secondary hyperparathyroidism that additional intake of vitamin $\mathrm{D}$ resulted in significant reductions in renin and angiotensin II plasma levels (18). Rats with chronic renal failure treated three times a week for eight weeks with vitamin D showed a decrease in renin, angiotensin renin receptor and vascular growth factor mRNA.

According to Li (2002) vitamin D deficiency is associated with an increased risk of cardiovascular disease, both in healthy subjects and in patients at risk. An important mark and a factor predicting future cardiovascular risk is the rigidity of the arterial wall. In patients with chronic renal and heart disease, it was found that low concentrations of $25(\mathrm{OH}) \mathrm{D}$ and 1,25 $(\mathrm{OH}) 2 \mathrm{D} 3$ were associated with increased arterial rigidity. The probable mechanism by which vitamin D levels affect cardiovascular risk is through the renin-angiotensin system. Low plasma levels of the vitamin may stimulate the renin-angiotensin system in healthy individuals $(31,32)$.

In addition, results from Sugden's research (2008) show that left ventricular hypertrophy is one of the most common complications in cardiovascular disease and chronic kidney disease, with the pathophysiological mechanisms leading to its development being multifactorial, but one of these factors is vitamin $\mathrm{D}$ deficiency. Schleithoff and colleagues reported improving the profile of cytokines in patients with congestive heart failure after vitamin D therapy. The predicted mechanism of vitamin D-associated cardioprotection is the suppression of the activity of the renin-angiotensin system (35).

According to Vaidya (2012) in patients with angina are found significantly lower levels of $25(\mathrm{OH})$ vitamin $\mathrm{D}$ in serum than those with abnormal controls and this factor is being independent of the degree of ischemia and the presence of hypertension.

Another predictor of cardiovascular mortality described in Barrett 's studies (2017) is vascular calcification, which is common in patients with chronic kidney disease, with atherosclerosis and arteriosclerotic vascular changes being the main implications. Impaired calcium phosphate metabolism induces endothelial dysfunction, vascular smooth muscle calcification, insulin resistance, activation of the renin-angiotensin system. Vitamin D suppresses foaming cells and cholesterol penetration into macrophages by stimulating their differentiation and activating cholesterol 7-alpha-hydroxylase and capturing low-density lipoproteins. By suppressing parathyroid hormone, vitamin $\mathrm{D}$ restores the activity of osteoblasts and osteopenic expression, inactivates the renin-angiotensin system and suppresses TGF-beta1-tubular interstitial fibrosis (36).

Vaidya (2012) suggests that in patients with chronic kidney disease, in parallel with a gradual decrease in levels of $1,25(\mathrm{OH})_{2}$ Vitamin D, the level of erythropoietin is also reduced and the renin-angiotensin system is activated, with the main consequences of anemia and secondary hyperparathyroidism (32). 
In the role of a multifunctional hormone and neutralizing the activity of the reninangiotensin system, vitamin $\mathrm{D}$ can stabilize body homeostasis and, in particular, the normal physiological functions of the kidneys and the cardiovascular system.

\section{REFERENCES}

1. Arita D, Reis R, Pessoa B, Casarini D. Renin-angiotensin system: old system with new different components, New aspects of the renin-angiotensin system, chapter 1:314, 2016.

2. Bonnet F, Candido R, Carey RM, Casley D, Russo LM, Osicka TM, Cooper ME and Cao Z. Renal expression of angiotensin receptors in long-term diabetes and the effects of angiotensin type 1 receptor blockade, Journal of Hypertension, 20(8):1615-24, 2002.

3. Borghi $\mathrm{C}$ et al. Renin-angiotensin system at the crossroad of hypertension and hypercholesterolemia, Nutrition, Metabolism and Cardiovascular Diseases 2017; 27:115-120.

4. Burns K. Angiotensin II and its receptors in the diabetic kidney, American Journal of Kidney Diseases, 36(3):449-467, 2000.

5. Carey RM, Wang Z-Q, Siragy HM. Role of the angiotensin type 2 receptor in the regulation of blood pressure and renal function. Hypertension, 35:155-63, 2000.

6. Carey RM. Cardiovascular and Renal Regulation by the Angiotensin Type 2 Receptor, Hypertension, 45:840-844, 2005.

7. De Mello W. Local Renin Angiotensin Aldosterone Systems and Cardiovascular Diseases, Medical Clinics of North America, 101:117-127, 2017.

8. Favre GA, Esnault VL. Modulation of glucose metabolism by the reninangiotensin-aldosterone system, Am J Physiol. Endocrinol. Metabol., 306(6):E435-449, 2015.

9. Giacchetti G, Sechi LA, Rilli S, Carey RM. The renin-angiotensin-aldosterone system, glucose metabolism and diabetes. Trends Endocrinol. Metab., 16:120-6, 2005.

10.Hsueh W and Wyne K. Renin-AngiotensinAldosterone system in diabetes and hypertension, J. Clin. Hypertens, 13:224237, 2011.

11.Cabandugama P, Gardner M. The Renin Angiotensin Aldosterone System in Obesity and Hypertension: Roles in the Cardiorenal Metabolic Syndrome, Medical Clinics of North America, 101:129-137, 2017.
12.Suzuki Y, Ruiz-Ortega M, Egido J. Angiotensin II: A double-edged sword in inflammation. $J$ Nephrol, 13(Suppl 3):S101-S110, 2000.

13.Patel S, Rauf A. Renin-angiotensinaldosterone (RAAS): The ubiquitous system for homeostasis and pathologies, Biomedicine and Pharmacotherapy, 94:317-325, 2017.

14.Touyz RM, Berry C. Recent advances in angiotensin II signaling, Braz. J. Med. Biol. Res., 35(9):1001-15, 2002.

15.Rahimi Z, Moradi M, Nasri H. A systematic review of the role of reninangiotensin aldosterone system genes in diabetes mellitus, diabetic retinopathy and diabetic neuropathy, Journal of Research in Medical Sciences, 19(11):1090-1098, 2014.

16.Agarwal R. Proinflammatory effects of oxidative stress in chronic kidney disease: role of additional angiotensin II blockade, Am. J. Physiol. Renal. Physiol., 284:F8639, 2003.

17. Arendshorst W, Brannstrom K, Ruan X. Actions of angiotensin II on the renal microvasculature, J. Am. Soc. Nephrol., 10:5149-61, 1999.

18. Basit S. Vitamin D in health and disease: a literature review, Br J Biomed Sci, 70:161172, 2013.

19.Drechsler C, Pilz S, Verduijn M, Tomaschitz A, Krane V, Espe K, Dekker F, Ritz E, Wanner, C. Vitamin D deficiency is associated with sudden cardiac death, combined cardiovascular events, and mortality in hemodialysis patients, Eur Heart J, 31:2253-2261, 2010.

20.Heaney RP. Vitamin D in health and disease, Clin J Am Soc Nephrol, 3:15351541, 2008.

21.Holick MF. Vitamin D deficiency, $N$ Engl $J$ Med, 357:266-281, 2007.

22. Autier P, Gandini S. Vitamin D supplementation and total mortality, Arch Intern Med, 167:1730-1737, 2007.

23. Yilmaz H, Kaya M, Sahin M, Delibasi T. Is vitamin D status a predictor of glycaemic regulation and cardiac complication in type 2 diabetes mellitus patients?, Diabetes \& Metabolic syndrome: Clinical research and reviews, 6:28-31, 2012.

24.Sipahi S, Acikgoz S, Genc A, Yildirim M, Solak Y, Tamer A. The association of vitamin $\mathrm{D}$ status and vitamin $\mathrm{D}$ replacement therapy with glycemic control, serum uric acid levels, and microalbuminuria in patients with type 2 diabetes and chronic 
kidney disease, Med Princ Pract, 26:146151, 2017.

25.Atalay E, Kivrak Y. The possible effect of vitamin $\mathrm{D}$ on uric acid levels in diabetic patients, World Clin J Med Sci, 1(2):77-83, 2017.

26. Mostafa D, Nasra R, Zahran N, Ghoneim M. Pleiotropic protective effects of vitamin D against high fat diet-induced metabolic syndrome in rats: one for all, European journal of pharmacology, 792:38-47, 2016.

27.Zittermann A. Vitamin D in preventive medicine: are we ignoring the evidence? $\mathrm{Br}$ J Nutr, 89:552-572, 2003.

28. Holick MF. Vitamin D: importance in the prevention of cancers, type 1 diabetes, heart disease, and osteoporosis. Am J Clin Nutr, 79: 362-371, 2004.

29. Subih H, Al-Tamami H. Decreased weight gain and enhanced serum biochemical parameters in rats after vitamin $\mathrm{D}$ and $\mathrm{Ca}$ supplementation, Mal J Nutr, 24(2):251256, 2018.

30.Li YC. Vitamin D regulation of the reninangiotensin system, $J$ Cell Biochem, 88(2):327-33, 2003.

31.Li YC, Kong J, Wei M, Chen ZF, Liu S, Cao L. 1,25-dihydroxy vitamin $\mathrm{D}(3)$ is a negative endocrine regulator of the reninangiotensin system, J Clin Invest, 110:229238, 2002.

32.Vaidya A, Williams JS. The relationship between vitamin $\mathrm{D}$ and the reninangiotensin system in the pathophysiology of hypertension, kidney disease, and diabetes, Metabolism, 61(4)450-458, 2012.

33. Chiu KC, Chu A, Go VLW. Hypovitaminosis D is associated with insulin resistance and b cell dysfunction. Am J Clin Nutr, 79: 820-825, 2004.

34.Chandel N, Ayasolla K. Vitamin D receptor deficit induces activation of the reninangiotensin system via SIRT1 modulation in podocytes, Experimental and Molecular Pathology, 102:97-105, 2017.

35.Sugden J, Davies J, Witham M, Morrist A, Struthers A. Vitamin D improves endothelial function in patients with type 2 diabetes mellitus and low vitamin D levels, Diabetes UK, Diabetic Medicine, 25:320325, 2008.

36.Barrett T. Type 2 diabetes mellitus: incidence, management and prognosis, Paediatrics and Child Health, 27:166-170, 2017. 